\title{
Robotic first rib resection for thoracic outlet syndrome
}

\author{
Farid Gharagozloo, Nabhan Atiquzzaman, Mark Meyer, Barbara Tempesta, Scott Werden
}

Center for Advanced Thoracic Surgery, Global Robotics Institute, Advent Health Celebration, University of Central Florida, Celebration, FL, USA Contributions: (I) Conception and design: F Gharagozloo, N Atiquzzaman, M Meyer; (II) Administrative support: B Tempesta; (III) Provision of study materials or patients: F Gharagozloo, N Atiquzzaman, M Meyer; (IV) Collection and assembly of data: B Tempesta; (V) Data analysis and interpretation: F Gharagozloo, N Atiquzzaman, M Meyer; (VI) Manuscript writing: All authors; (VII) Final approval of manuscript: All authors. Correspondence to: Farid Gharagozloo, MD. Center for Advanced Thoracic Surgery, Global Robotics Institute, Advent Health Celebration, University of Central Florida, 400 Celebration Place, Celebration, FL 34747, USA. Email: Farid.Gharagozloo.MD@AdventHealth.com.

\begin{abstract}
Robotic resection of the "offending portion" of the first rib in patients with thoracic outlet syndrome (TOS) has been associated with excellent results. The results have been due to (I) a better understanding of the pathogenesis of TOS, and (II) the technical advantages of the robotic platform. This article outlines the recent understanding of the pathogenesis of TOS, and reports the experience with robotic resection of the "offending portion" of the first rib in patients with neurogenic and venous TOS. Patients diagnosed with TOS underwent robotic first rib resection. Diagnosis of TOS was made by magnetic resonance angiography (MRA). On a thoracoscopic platform, the robot was used to dissect the "offending portion" of the first rib. A total of 162 patients underwent robotic first rib resection. Eighty-three patients underwent robotic first rib resection for Paget-Schroetter syndrome (PSS) (venous TOS). There were 49 men and 34 women. Mean age was $24 \pm 8.5$ years. Operative time was $127.6 \pm 20.8$ minutes. Median hospitalization was 4 days. There were no surgical complications, neurovascular injuries, or mortality. At a median follow-up of 24 months, all patients had an open subclavian vein (SV) for a patency rate of $100 \%$. Seventy-nine patients underwent robotic first rib resection for neurologic symptoms of the upper extremity (neurogenic TOS). There were 29 men and 50 women. Mean age was $34 \pm 9.5$ years. Operative time was $87.6 \pm 10.8$ minutes. There were no intraoperative complications. Hospital stay ranged from 2-4 days with a median hospitalization of 3 days. There were no neurovascular complications. There was no mortality. In patients with neurogenic symptoms, Quick DASH Scores (mean \pm SEM) decreased from $60.3 \pm 2.1$ preoperatively to $5 \pm 2.3$ in the immediate postoperative period, and $3.5 \pm 1.1$ at 6 months $(\mathrm{P}<0.0001)$. Immediate relief of symptoms was seen in $71 / 79$ (91\%) patients. Persistent paresthesia was seen in $9 / 79(9 \%)$ immediately postop and $3 / 79(3.8 \%)$ patients at 6 months. Following the appropriate identification of the "offending portion" of the first rib which results in compression of the SV at its junction with the innominate vein by MRA, robotic resection of the "offending portion" of the first rib allows is associated with excellent results.
\end{abstract}

Keywords: Robotic first rib resection; thoracic outlet syndrome (TOS); neurogenic thoracic outlet syndrome (neurogenic TOS); Paget-Schroetter syndrome (PSS); subclavian vein compression (SV compression)

Submitted Feb 25, 2020. Accepted for publication Aug 07, 2020.

doi: $10.21037 /$ jtd-2019-rts-04

View this article at: http://dx.doi.org/10.21037/jtd-2019-rts-04

\section{Introduction}

In 1956, Peet attempted to unify a group of diverse patients with symptoms in the shoulder and upper extremity who presented with pain, numbness, tingling, and swelling, under the umbrella of neurogenic "thoracic outlet syndrome" ("TOS") (1). Since that publication, which divided TOS into arterial, venous, and neurogenic varieties, TOS has 
been associated with a number of controversies. These have included confusion about the actual pathophysiology of the disease, the appropriate diagnostic tests, and the appropriate therapeutic interventions. In the six decades since Peet's publication, the only consistent aspects of TOS have been the confusion among medical practitioners, difficulty in making the diagnosis, and the poor results with surgical intervention. Furthermore, if the purpose of unifying the group of patients with neurovascular symptoms of the upper extremity was to improve therapeutic outcomes, based on the published experience, such an effort has not been successful $(2,3)$.

A discussion about the role of robotic first rib resection in the treatment of TOS needs to start with an understanding of the historic background which is responsible for the classification of TOS, which to a large extent has given rise to the complexities in terms of definition, diagnosis, and therapy which face the surgeon in the modern age.

\section{Historical perspective}

The cervical rib was first described in $150 \mathrm{AD}$ by Galen, the Greek anatomist and court physician to Marcus Aurelius. For almost the next two millennia, the symptomatic manifestations of cervical ribs were unknown. In 1818, Sir Cooper, described a young woman with pulselessness and gangrenous spots on her fingers in conjunction with a palpable hard mass at the base of the neck. He attributed the patient's symptoms to "a projection of the lower cervical vertebra towards the clavicle, and consequent pressure upon the subclavian artery" (4). Cooper referred to this condition as "cervical rib syndrome" (CRS). In 1861, Coote, an English surgeon, performed the first surgical procedure for CRS. Interestingly, Coote described the same trepidation that is experienced by modern surgeons in treating cervical rib syndrome: "But the region was not a pleasant one for any proceeding demanding the use of the knife. The subclavian artery and vein were in front; the axillary plexus of nerves lay spread out above; below, the apex of the lung, covered by the pleura, rose up in dangerous proximity; on the scalenus was the phrenic nerve; while towards the mesial line were the important vessels and nerves passing to the head, together with the vertebral vessels and thoracic duct. You can understand, therefore, why I was cautious in what I did" (5).

In 1869 , Gruber published a classification system for cervical ribs. His classification was based on the length of the cervical rib and its attachment to the first rib. The "Gruber Classification" remains in use today (6).
Until 1902, CRS was thought to affect only the subclavian artery (SA). Up to that time, CRS was defined as compression of the second portion of the SA in the neck by an abnormal cervical rib or associated fibrous bands. That year, for the first time, Buzzard described seven patients with "neurologic" symptoms of the upper extremity whose symptoms were attributed to compression of the brachial plexus by a cervical rib (7). Thereafter, "CRS" was defined as: neurovascular symptoms in the upper extremity which resulted from compression of the SA or the brachial plexus in the presence of a cervical rib (8). The diagnosis of CRS was facilitated by the advent of X-ray technology in the turn of the twentieth century. For the first time, prior to surgical intervention, $\mathrm{X}$-rays provided an objective means of imaging and recognizing cervical ribs.

On the other hand, X-ray technology also resulted in some more confusion, as there were many patients who had upper extremity neurovascular symptoms like those in patients with CRS but without a cervical rib on $\mathrm{X}$-ray. This gave rise to the curious concept of "cervical rib syndrome without a cervical rib"! In 1910, Murphy of Australia performed the first resection of a "normal first rib" in a patient who presented with upper extremity "nerve related symptoms" without a visible cervical rib on X-ray. Intraoperatively he observed a broad insertion of the anterior scalene muscle onto the first rib at the anterior scalene tubercle located on the medial aspect of the first rib (9). Although this area was far away from the brachial plexus, he removed the insertion and a small part of the first rib hypothesizing that somehow the patient's neurologic symptoms in the upper extremity were related to compression by the hypertrophied scalene muscle. Interestingly the patient experienced some but not complete relief in symptoms. In the following years a number of investigators attributed nerve related symptoms in the upper extremity in the absence of a cervical rib to compression of the brachial plexus caused by upward elevation of the first rib by a hypertrophied anterior scalene muscle and coined the term: "scalenus anticus syndrome" $(10,11)$. As Scalenectomy alone was associated with poor results, eventually resection of the first rib along with scalenectomy was advocated for these patients (12). At the same time, due to a lack of radiographic findings in these patients, many attempts were made to diagnose the "compression" by clinical maneuvers such as Adson's and Wright tests $(13,14)$. Invariably these maneuvers had a poor sensitivity for the diagnosis of the underlying pathology, and poor predictive value for surgical success (14). 


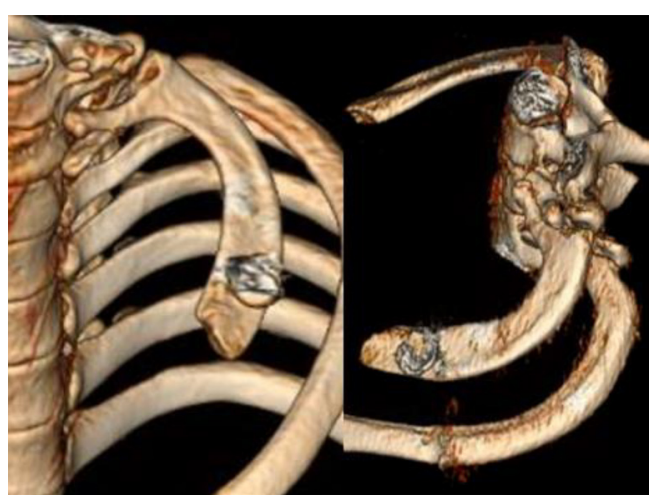

Figure $13 \mathrm{D}$ reconstruction of computerized axial tomograms in patients with PSS demonstrates the presence of a congenitally malformed bony tubercle which forms a tighter and wider joint at the junction of the first rib and the sternum. 3D, threedimensional; PSS, Paget-Schroetter syndrome.

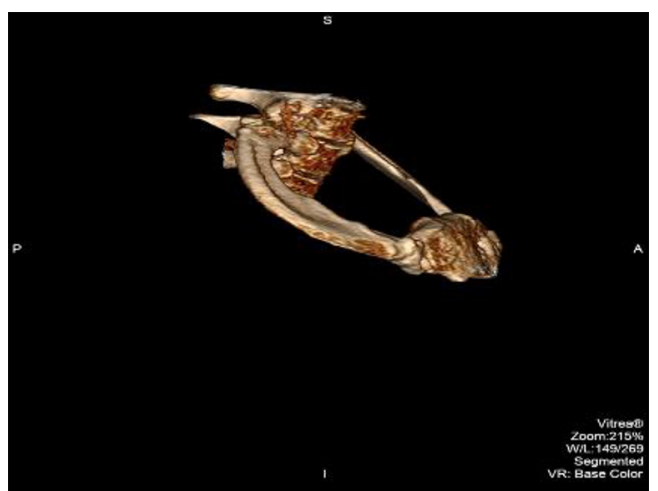

Figure $23 \mathrm{D}$ reconstruction of computerized axial tomograms in patients with PSS demonstrates the wider and less mobile coststernal joint which "locks" the medial aspect of the first rib into place and results in extrinsic compression of the boney tubercle onto the subclavian vein at its junction with the innominate vein. 3D, three-dimensional; PSS, Paget-Schroetter syndrome.

Although the majority of patients presented with nerve related symptoms in the upper extremity, the concept of neurovascular compression due to upward elevation of the first rib by a hypertrophied anterior scalene muscle and compression of neurovascular structures in the thoracic outlet was further reinforced by reports of patients who presented with thrombosis of the subclavian vein (SV), Paget-Schroetter syndrome (PSS). In 1875 and 1884, Sir Paget and von Schroetter first described patients with thrombosis of the SV which was precipitated with excessive activity of the upper extremity $(15,16)$. Later in the 1940's Hughes and Illig coined the terms PSS and effort thrombosis, and made a connection to compression of the $\mathrm{SV}$ in the thoracic outlet $(17,18)$.

In the twentieth century, conditions associated with neurovascular symptoms of the upper extremity have been called: brachial compression neuritis, scalenus anticus syndrome (Naffziger syndrome), costoclavicular syndrome/ costoclavicular compression syndrome, hyperabduction syndrome/Wright syndrome, brachiocephalic syndrome, brachiocephalic vascular syndrome, nocturnal paresthetic brachialgia, brachialgia statica paresthetica, PSS/effort thrombosis of the SV, axillary-subclavian vein thrombosis (ASVT), cervicobrachial syndrome, first thoracic rib syndrome, superior outlet syndrome, fractured claviclerib syndrome, cervical rib and band syndrome. Clearly the vast array of classifications points to an overall lack of understanding of the underlying disease processes.

In the near six decades since Peet's report, confusion about TOS and the poor results with surgical intervention have continued. During this time, a great deal of attention has been focused on the surgical approach to the resection of the first rib and whether other procedures such as scalenectomy, neurolysis, and pectoralis minor tenotomy should be included with first rib resection. However, irrespective of the approach and the specifics of the surgical procedures, the results of surgery have been disappointing $(19,20)$.

\section{Pathogenesis of PSS}

Recently, examination of the medial aspect of the resected first ribs in patients with PSS has demonstrated the presence of a congenitally malformed bony tubercle which forms a tighter and wider joint at the junction of the first rib and the sternum (21). In a number of patients with a wider tubercle there is even a second pseudarthrosis with the head of the clavicle $(22,23)$. It has been observed that the wider and less mobile cost-sternal joint "locks" the medial aspect of the first rib into place and results in extrinsic compression of the boney tubercle onto the SV at its junction with the innominate vein. Therefore, the abnormal medial aspect of the first rib compresses the $\mathrm{SV}$ at the "thoracic outlet". This abnormal tubercle on the medial aspect of the first rib can be seen on three-dimensional (3D) reconstruction of computerized axial tomograms in patients with PSS (Figures 1-3). In addition, the extrinsic compression of the SV by the tubercle at the medial aspect of the first rib can be demonstrated on dynamic 


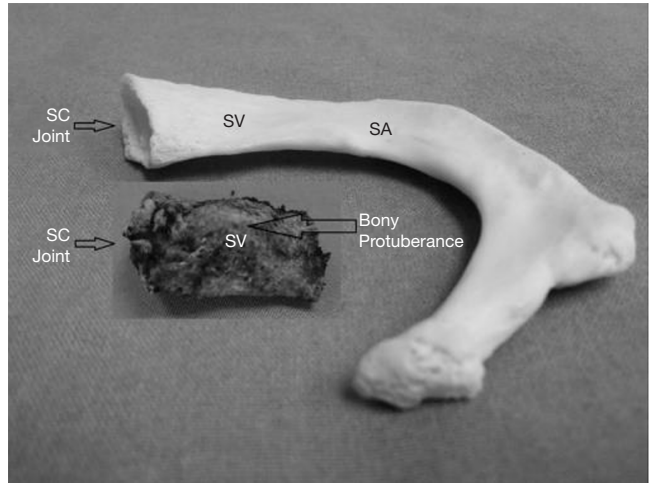

Figure 3 Comparison of the abnormal bony tubercle from a patient with PSS with the medial aspect of a normal rib. The normal rib has a groove for the SV and SA. The bony tubercle forms a tighter SC joint and compresses SV as it crosses over the first rib. PSS, Paget-Schroetter syndrome; SV, subclavian vein; SA, subclavian artery; SC, costo-sternal.

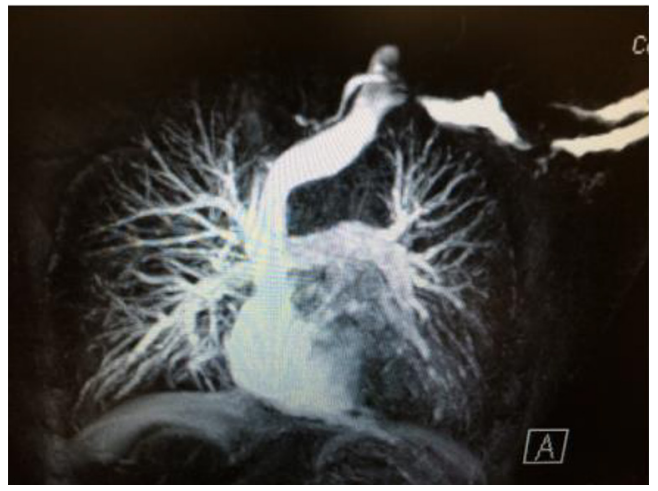

Figure 4 MRA with elevation of the left upper extremity shows compression of the SV by the abnormal bony tubercle in a patient with PSS. MRA, magnetic resonance angiography; SV, subclavian vein; PSS, Paget-Schroetter syndrome.

magnetic resonance angiography (MRA) with maneuvers and dynamic venography (Figures 4-6). Furthermore, these studies clearly demonstrate that the SV compression increases with elevation of the arm above the shoulder. It can be surmised that without the benefit of the sophisticated modern imaging, and relying only on intraoperative observations, it is likely that historically surgeons have erroneously referred to this tubercle as the hypertrophied costoclavicular ligament, and the hypertrophied scaleneus anticus tubercle. In patients with PSS, Gharagozloo et al. have demonstrated that disarticulation of the costosternal (SC) joint and resection of the "offending portion"

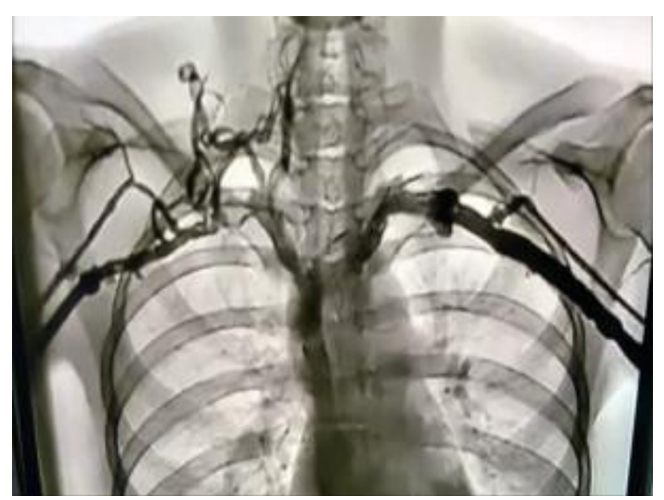

Figure 5 Venogram of a patient with PSS. With the arms in the adducted position there is flow through the SVs. The right SV is compressed more than the left and shows collateral formation. PSS, Paget-Schroetter syndrome; SV, subclavian vein.

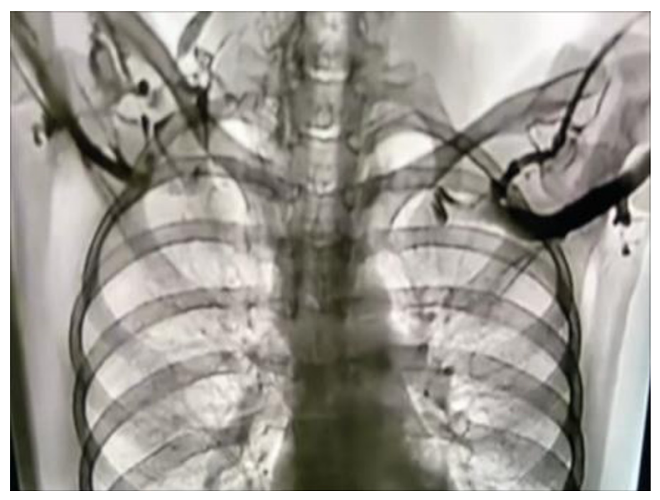

Figure 6 Venogram of a patient with TOS. With the arms in the elevated position the SVs are occluded bilaterally, with cessation of flow. TOS, thoracic outlet syndrome; SV, subclavian vein.

of the first rib (portion of the rib medial to the SA) results in decompression of the SV $(22,23)$. Furthermore, these authors have reported that preoperative dynamic MRA has been demonstrated to have a predictive value of $100 \%$ for surgical success in patients with PSS. They have suggested that the medial aspect of the first rib which compresses the SV at its junction with the innominate vein should be called: the "offending portion" of the first rib and this syndrome should be referred to as "subclavian vein compression syndrome".

In addition, dynamic MRA in patients with PSS has demonstrated the presence bilateral disease in the head of the first rib (Figure 7). This observation further clarifies reports of PSS affecting the contralateral extremity, and supports the congenital pathogenesis of the disease. 


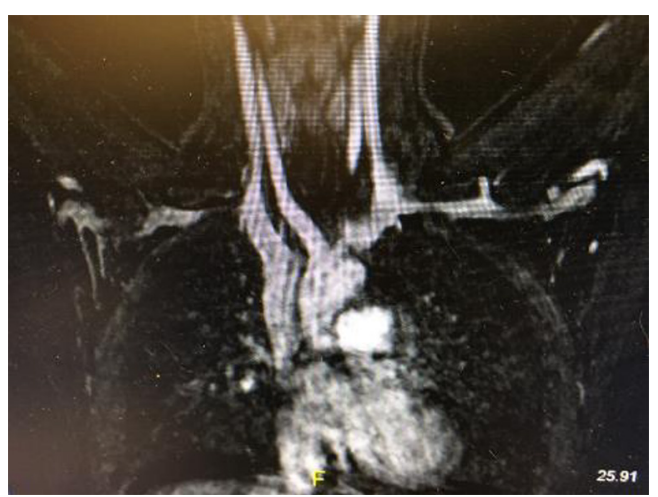

Figure 7 MRA with elevation of the left upper extremity in a patient who has had a transthoracic first rib resection on the right for "neurogenic" TOS. There is residual extrinsic compression of the right SV from the retained SC joint. In addition, the left $\mathrm{SV}$ also shows extrinsic compression at its junction with the innominate vein pointing to the bilateral and congenital nature of the disease. MRA, magnetic resonance angiography; TOS, thoracic outlet syndrome; SV, subclavian vein; SC, costo-sternal.

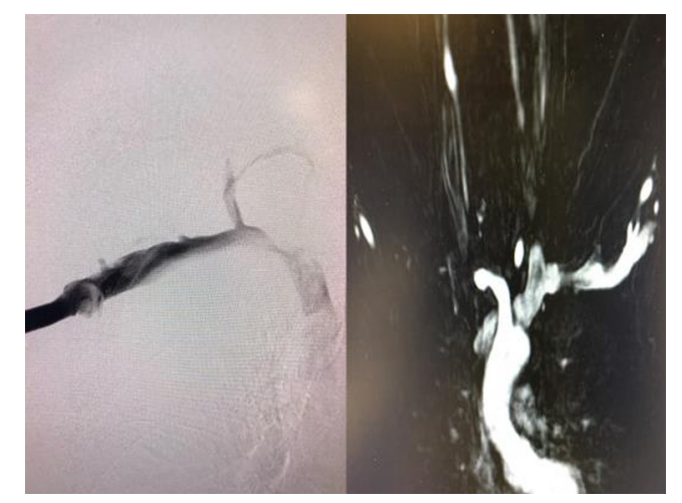

Figure 8 Preoperative MRA and venogram with elevation of the left upper extremity in a patient who has had a transthoracic first rib resection on the right for "neurogenic" TOS. There is compression of the subclavian innominate junction. MRA, magnetic resonance angiography; TOS, thoracic outlet syndrome.

Therefore, it has been suggested that PSS results from a congenital malformation of the first rib which compresses the SV in the thoracic outlet, and with prolonged compression stemming from activities that elevate the arm above the shoulder, result in thrombosis of the vein.

\section{Pathogenesis of neurogenic TOS}

A study of patients with neurogenic TOS who had

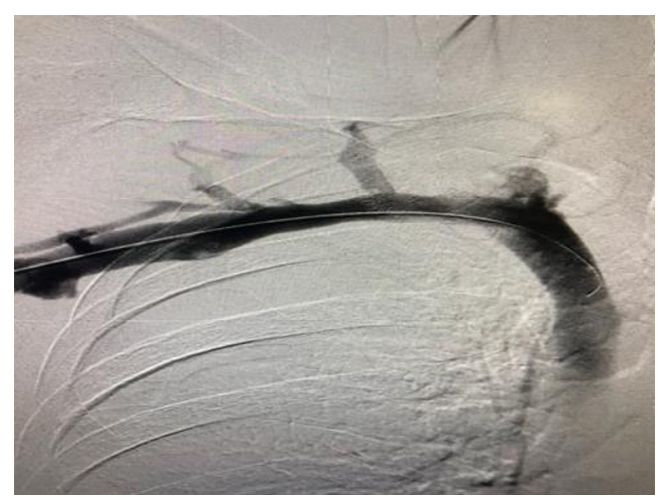

Figure 9 Postoperative (following robotic resection of the residual first rib at the SC junction) venogram with elevation of the left upper extremity in a patient who has had a transthoracic first rib resection on the right for "neurogenic" TOS. The SV is completely patent and the patient experienced complete resolution of the "neurogenic" symptoms. SC, costo-sternal; TOS, thoracic outlet syndrome; SV, subclavian vein.

persistent upper extremity pain following first rib resection by the transaxillary and supraclavicular approaches, revealed persistent extrinsic compression of the subclavian innominate junction on dynamic MRA. These patients underwent video-assisted exploration of the chest which showed a persistent SC joint despite evidence for prior removal of the first rib. Disarticulation of the cost-sternal joint and removal of the remaining portion of the first rib which bore a tubercle similar to that which was seen in patients with PSS, alleviated the extrinsic compression of the subclavian-innominate vein junction on postoperative dynamic MRA and resulted in relief of Neurogenic symptoms in all patients (Figures 8,9). Based on this observation, it was hypothesized that Neurogenic TOS may be the manifestation of nerve pain which results from venous compression and the resultant venous ischemia of the nerves in the upper extremity. This hypothesis is based on the fact that the upper extremity is fed by a single artery and vein as an "end organ". In such a setting, studies have demonstrated that the blood-nerve barrier in the nerve root was more easily broken by venous congestion than by arterial ischemia. Venous congestion may be an essential factor precipitating circulatory disturbance in nerve roots and inducing neurogenic intermittent claudication (24). Therefore, compression of the SV at its junction with the innominate vein may result in elevation of venous pressure, a decrease in arterial flow, and relative ischemia of the nerves of the upper extremity. Venous ischemia of the upper 
extremity nerves may manifest as pain, tingling, paresthesia and numbness, and varying degrees of neurogenic intermittent claudication depending of the degree and duration of venous compression. Elevation of the extremity above the shoulder, may result in greater compression of the $\mathrm{SV}$, further venous congestion, further decrease in arterial flow, greater degree of ischemia of the upper extremity nerves, and exacerbation of symptoms. This phenomenon is demonstrated on dynamic magnetic resonance imaging (MRI) and venography. The pathophysiology of nerve pain in this setting has been likened to symptoms that result from "crossing one leg over the knee".

Only $5 \%$ of patients with the diagnosis of neurogenic TOS are found to have a cervical rib and are best classified as cervical rib syndrome. Ninety-five percent of patients with the diagnosis of neurogenic TOS are believed to have neurologic manifestations of upper extremity ischemia and compression of the SV by an abnormal first rib at the thoracic outlet. Therefore, it was hypothesized that robotic transthoracic resection of the medial aspect of the first rib at the SC junction in patients of neurogenic TOS diagnosed by MRA will result in relief of symptoms. In a proof of concept study, surgical outcomes in patients diagnosed with neurogenic TOS who underwent robotic first rib resection were reviewed. Diagnosis was made by history, physical exam, MRI of C-spine, orthopedic and neurologic examination, nerve conduction studies, and MRA of the thoracic outlet with maneuvers. Patients with cervical ribs or cervical bands were excluded.

Patients with compression of the SV by the medial aspect of the first rib ("the "offending portion") underwent robotic resection of the first rib and were the subjects of this study. Subjective symptoms were assessed by Disabilities of the Arm, Shoulder and Hand Questionnaire Score (Quick DASH), 1 week, 1 month and 6 months. The Quick DASH is an abbreviated version of the original DASH outcome measure. In comparison to the original 30 items DASH outcome measure, the Quick DASH only contains 11 items. It is a questionnaire that measures an individual's ability to complete tasks, absorb forces, and severity of symptoms. The Quick DASH tool uses a 5-point Likert scale from which the patient can select an appropriate number corresponding to his/her severity level/function level $(25,26)$. The extrinsic compression of the SV on postoperative MRA and angiogram with maneuvers was assessed at 1 month. In this proof of concept study, patients with Neurologic symptoms of the upper extremity who were classified as "neurogenic TOS" had complete relief of symptoms with relief of compression of the SV after robotic resection of the medial aspect of the first rib and disarticulation of the SC joint.

Removal of the medial aspect of the first rib and decompression of the thoracic outlet resulted in relief of upper extremity neurovascular symptoms in greater than $97 \%$ of patients who presented with neurogenic symptoms in the upper extremity.

These observations have led these investigators to propose the "ischemic" versus the "compressive" mechanism for neurogenic TOS. They have likened neurogenic TOS in the upper extremity to the well-known phenomenon of paresthesia, numbness, and pain which results from crossing one leg over the other at the knee. Despite commonly held belief that "crossing leg" syndrome results from compression of the peroneal nerve, it has been shown that, in fact, it is the result of the compression of the popliteal vein by the contralateral knee.

The present understanding of TOS is that it is the manifestation of a congenital malformation of the first rib. The congenital malformation is in the form of a pronounced tubercle which results in an abnormal costoclavicular-sternal joint and the compression of the SV at its junction with the innominate vein. The congenital disease is bilateral with variable symptomatic expression. The compression of the SV in the thoracic outlet results in a spectrum of disease which ranges from neurologic symptoms resulting from venous ischemia of the upper extremity nerves (Peet's neurogenic TOS) to thrombosis of the SV with prolonged compression (Paget-Schroetter or Peet's venous TOS). Neurogenic and venous TOS appear to be caused by the same pathophysiologic process which compresses the $\mathrm{SV}$ at its junction with the innominate vein. They represent different clinical manifestations of the same disease and represent the spectrum from pain to venous thrombosis. It is suggested that these conditions are best classified "subclavian vein compression syndrome".

\section{Rethinking "TOS"}

Peet's classification of TOS was based on anatomic rather than symptomatic presentation of the disease. Based on recent studies, in order to decrease confusion and to improve therapeutic results with TOS, the disease should be classified based on the underlying pathologic entity. Acquired and traumatic abnormalities of the clavicle and first rib should be classified separately.

Clearly after the more common and objectively 
supported diagnoses of conditions that result in neurovascular symptoms of the upper extremity such as cervical spine disease, carpal tunnel disease and nerve entrapment syndromes, have been ruled out, there remains a group of patients who are suspected of having TOS. In these patients rather than the more usual classification such as arterial, venous, or neurogenic, the more accurate approach from a diagnostic and therapeutic approach is to classify them as:

(I) Cervical rib disease. In these patients an abnormal cervical rib or the associated bands that insert onto the first rib result in compression and displacement of the nerves or vessels in the neck. These patients can present with neurologic or vascular compromise. Patients with CRS can have complications relating to compression of the SA and the brachial plexus secondary to a well-formed cervical rib, or to an incompletely formed first rib, fibrous band associated with a rudimentary cervical rib, or a giant transverse process of C7. Although in the past, these patients have been classified as TOS, separation of these patients into CRS allows for a more precise diagnostic and therapeutic strategy and perhaps more importantly clears the way to a better understanding of diseases that result from anomalies of the first rib.

(II) Thoracic outlet disease. In these patients an abnormal first rib results in compression of the SV a the subclavian-innominate junction. Compression of the vein results in venous hypertension in the upper extremity and resultant neurologic symptoms. With prolonged compression of the subclavian-innominate junction, the vein clots giving rise to PSS. Therefore, patients who have been previously classified as neurogenic and venous TOS represent a variable symptomatic presentation of the same pathologic entity which affects the SV. The term arterial TOS should be abandoned as these patients are better classified under cervical rib disease or under traumatic causes. On the other hand, neurogenic and venous TOS appear to be caused by the same pathophysiologic process which compresses the SV at its junction with the innominate vein. They represent different clinical manifestations of the same disease and represent the spectrum from pain to venous thrombosis. It is suggested that these conditions are best classified "subclavian vein compression syndrome".

\section{Diagnosis of TOS}

Historically confusion about the pathogenesis of TOS has resulted in a number of diagnostic tests:

(I) Adson's test. In 1927, Adson and Coffey (27) described a technique to assess for evidence of circulatory symptoms caused by the presence of a cervical rib. "Diminution in volume of the radial pulse is common; the pulse can be decreased or obliterated by having the patient elevate the chin or rotate the head to the affected side while inspiring air". They believed that this evidence of circulatory disturbance warranted consideration for surgical resection of the cervical rib. Adson's test is associated with minimal inter-examiner reliability, is seen in normal individuals, and is inconclusive for the diagnosis of TOS.

(II) Wright's test. With a positive Wright's test, the radial pulse weakens or disappears when the arm is abducted and externally rotated. As with Adson's test, Wright's test is associated with minimal interexaminer reliability, is seen in normal individuals, and is inconclusive for the diagnosis of TOS.

(III) Neck radiographs.

(IV) Pulse volume recording.

(V) Cervical and supraclavicular dopplers.

(VI) CT of the neck and chest.

(VII) MRI of the neck.

(VIII) Nerve conduction studies.

(IX) Angiography.

(X) MRA of the thoracic outlet with arm maneuvers.

As a general rule, except for angiography and MRA of the thoracic outlet with arm maneuvers, the other tests have been inconclusive and are of historic value.

Presently MRA of the thoracic outlet with arm maneuvers is the test of choice in patients suspected of having TOS. This test shows the abnormal bony tubercle on the first rib with extrinsic compression of the subclavian innominate junction which is exacerbated with elevation of the arm above the shoulder. The predictive value of surgical success of this test in patients with Paget-Schroetter presentation is $100 \%$, and in patients with neurologic presentation is $97.3 \%$.

\section{Robotic first rib resection}

Surgical approaches to resection of the first rib have included transthoracic, transaxillary, supraclavicular, 


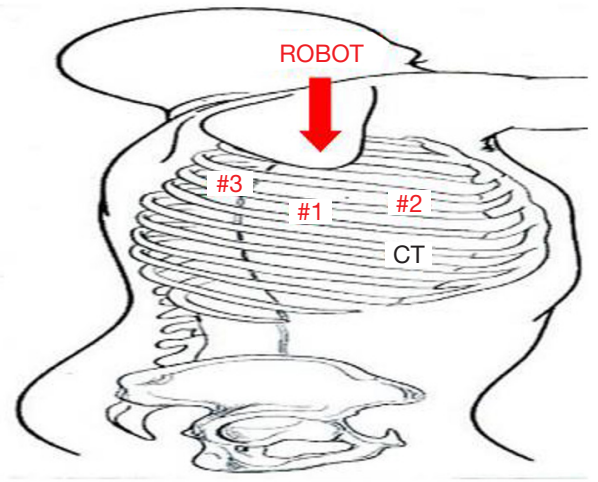

Figure 10 Patient positioning and trocar placement: three $2 \mathrm{~cm}$, non-trocar, incisions are made. In the right chest, incision \#1 is made at the 5 th IC space at the midaxillary line. Incision \#2 is made in the 4th IC space at the anterior axillary line. Incision \#3 is made in the 4th IC space at the posterior axillary line. A $1 \mathrm{~cm}$ incision (\#4) is made in the 6th IC space at the anterior axillary line. IC, intercostal.

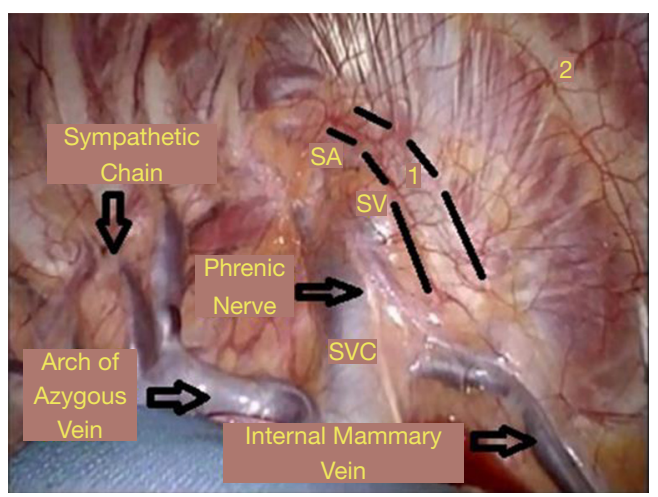

Figure 11 Phase I-VATS setup. Intraoperative photograph showing a retractor (Endopaddle Retract; Auto Suture, Covidien incorporated, Mansfield, MA, USA) which is used to retract the lung inferiorly. In patients with TOS the first rib is shorter and has a broader medial aspect due to the congenital abnormality of chest development. The positions of the SV and SA relative to the first rib are seen. TOS, thoracic outlet syndrome; SV, subclavian vein; SA, subclavian artery.

infraclavicular, and thoracoscopy. However, these approaches are associated with neurovascular complications, incomplete decompression of the SV and the medial aspect of the thoracic outlet, and incomplete resection of the most medial portion of the rib. These complications are in large part the result of the extrathoracic surgical approach and the difficulty with exposure and access. Theoretically, a minimally invasive transthoracic approach aimed at removing the offending compressive portion of the first rib would obviate the neurovascular complications and allow for complete resection of the offending portion of the first rib.

The robotic surgical systems have the advantages of $3 \mathrm{D}$ high-definition visualization, precise instrument maneuverability in a confined space.

\section{Surgical technique}

Due to the lack of availability of robotic end effectors designed for cutting the bone, presently the operation is performed on a video-assisted thoracoscopic surgery (VATS) platform. The robot is used to dissect the first rib, disarticulate the SC joint, and divide the scalene muscles. General anesthesia with single lung ventilation is used and patients are placed in the lateral decubitus position with the affected side up. A video of this procedure is available on https://youtu.be/1nImTFgG5TE.

\section{Phase I-VATS setup}

Three $2 \mathrm{~cm}$, non-trocar, incisions are made. In the right chest, Incision \#1 is made at the 5 th intercostal (IC) space at the midaxillary line. Incision \#2 is made in the 4th IC space at the anterior axillary line. Incision \#3 is made in the 4th IC space at the posterior axillary line. A $1 \mathrm{~cm}$ incision (\#4) is made in the 6th IC space at the anterior axillary line. The placement of incisions is in a mirror image configuration for the left chest (Figure 10). A retractor (Endopaddle Retract; Auto Suture, Covidien incorporated, Mansfield, MA, USA) is introduced through this incision and used to retract the lung inferiorly (Figure 11). At the end of the procedure a chest drain is inserted through this incision.

\section{Phase II-robot positioning and robotic dissection of the first rib}

The surgical robot (da Vinci, Intuitive Surgical, Inc., Sunnyvale, CA, USA) is positioned over the head of the patient. The camera is placed in incision \#1. For the placement of instruments, a 30-degree down-viewing camera is used. The right robotic arm with a hook cautery is positioned in incision \#2. The left robotic arm with a grasper is positioned in incision \#3. The assistant places a suction catheter through incision \#3 under the left robotic arm. A 30-degree up-viewing camera is used. The pleura 


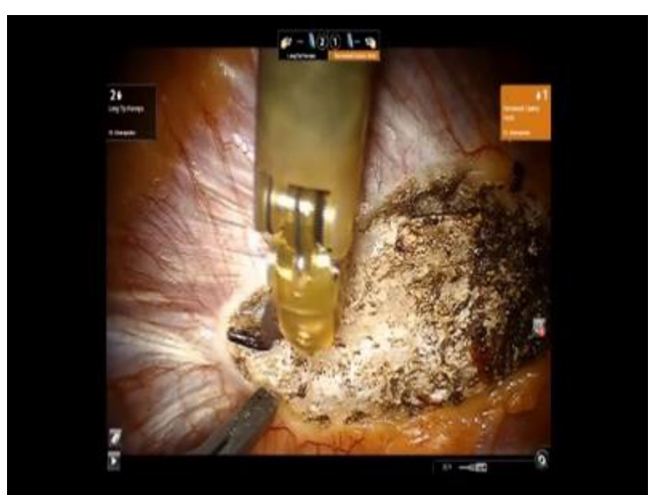

Figure 12 Phase II-robot positioning and robotic dissection of the first rib. View of the first rib through a 30-degree up viewing robotic camera. The hook cautery is used to clear the pleura on the underside of the rib.

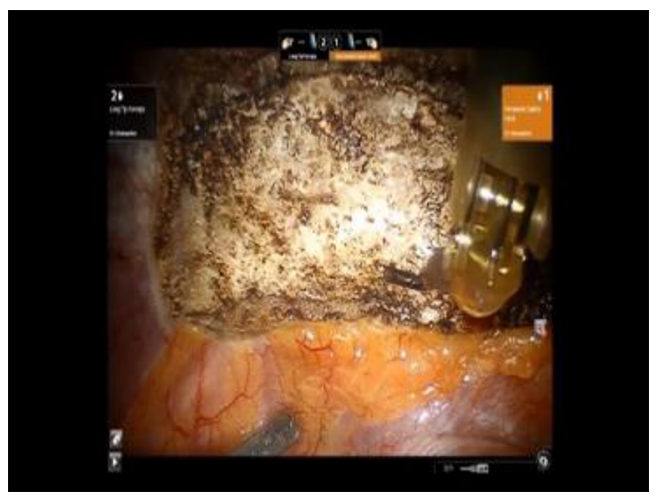

Figure 13 Phase II-robot positioning and robotic dissection of the first rib. View of the first rib through a 30-degree up viewing robotic camera. The edges of the rib are identified, as is the SC joint. Dissection of the pleura is carried just lateral to the SA. SC, costo-sternal; SA, subclavian artery.

overlying the first rib is dissected. The edges of the rib are identified, as is the SC joint. Dissection of the pleura is carried just lateral to the SA (Figures 12,13). The lateral and posterior aspect of the rib with the associated neurologic structure are left intact.

\section{Phase III-division of the first rib using VATS instruments}

Next, the robotic arms are withdrawn. A 30-degree VATS camera is introduced, and the rib under the SA is divided using a $6 \mathrm{~mm}$ thoracoscopic Kerrison bone cutter (Depuy Inc., Raynham, MA, USA) (Figures 14-17). This area

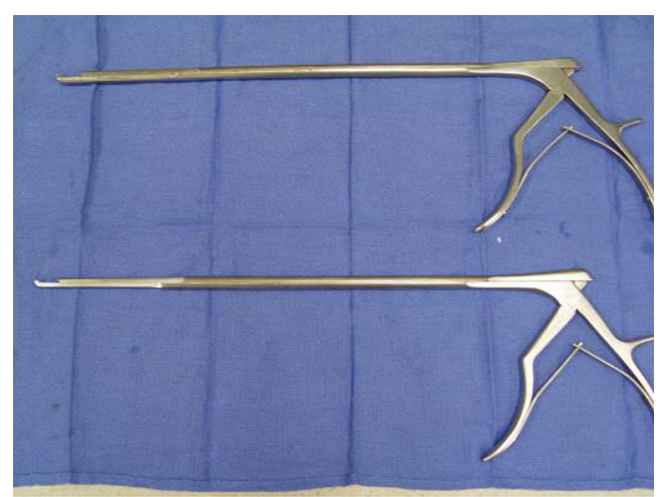

Figure 14 Phase III-division of the first rib using VATS instruments. Thoracoscopic Kerrison bone cutter (Depuy Inc., Raynham, MA, USA). VATS, video-assisted thoracoscopic surgery.

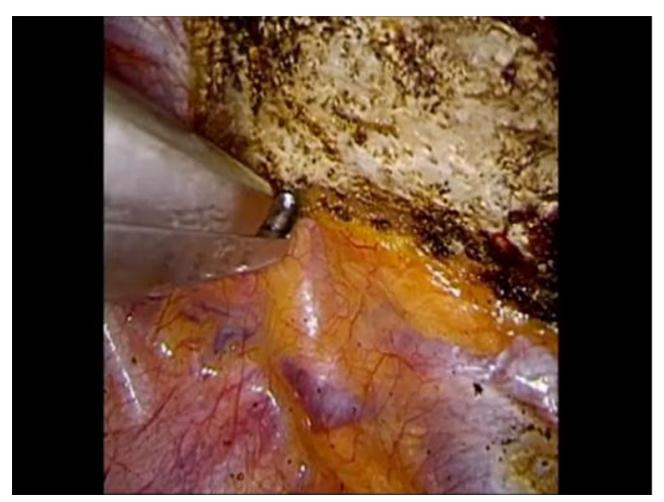

Figure 15 Phase III-division of the first rib using VATS instruments. The rib under the SA is divided using a $6 \mathrm{~mm}$ thoracoscopic Kerrison bone cutter. VATS, video-assisted thoracoscopic surgery; SA, subclavian artery.

represents the thinnest portion of the first rib and is most suitable for an osteotomy. The division of the rib at its midpoint allows for the rib to be pivoted on the SC and costovertebral joints in a trap door configuration.

\section{Phase IV-robotic dissection of the first rib and disarticulation of the SC joint}

The robotic arms are replaced in the same ports. A 30-degree down viewing robotic camera is introduced through incision \#1. A hook cautery is placed in the right robotic arm in incision \#2, and a second hook cautery is placed in the left robotic arm in incision \#3. The hook in the left arm is used to put downward traction on the rib as the hook cautery ( $30 \mathrm{cut} / 30$ coagulation setting) is used to 


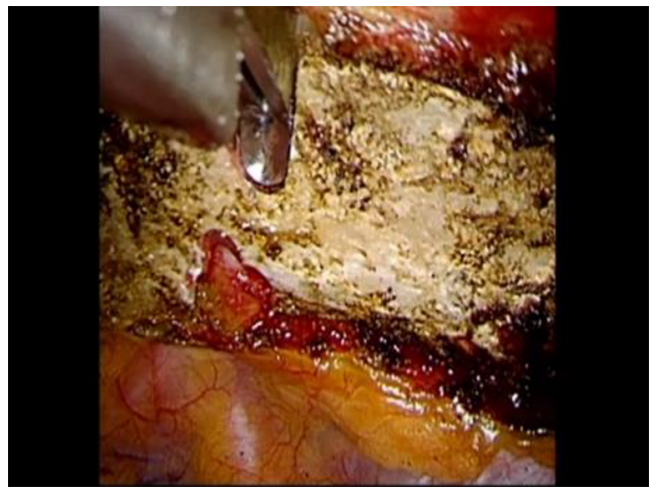

Figure 16 Phase III-division of the first rib. The $6 \mathrm{~mm}$ thoracoscopic Kerrison bone cutter is used to divide the rib just under the SA. The instrument is designed to protect the subclavian artery from injury. SA, subclavian artery.

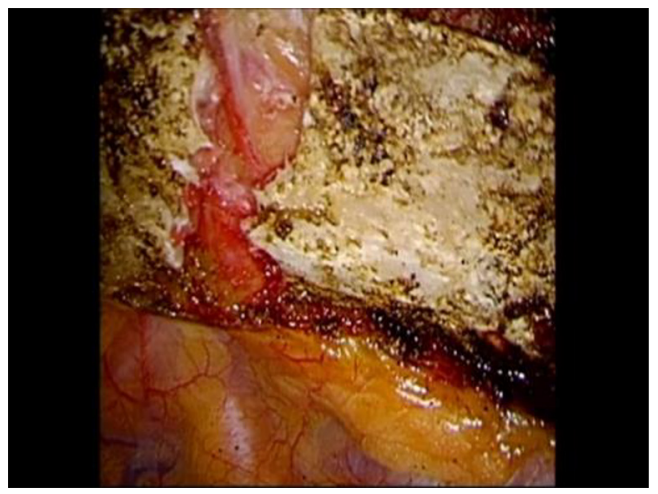

Figure 17 Phase III-division of the first rib using VATS instruments. The division of the rib at its midpoint allows for the rib to be pivoted on the SC and costovertebral joints in a trap door configuration. VATS, video-assisted thoracoscopic surgery; SC, costo-sternal.

dissect the first rib away from the SV, disconnect the scalene muscles from the rib, and disarticulate the rib from the sternal and times clavicular joint (Figures 18-21).

\section{Phase V-analgesia and chest closure}

Following the completion of the robotic procedure and undocking of the robot, the camera trocar is removed. An endoscopic camera (Olympus Endoeye 0 Degree) is introduced through the anterior port and used to visualize the paravertebral pleura. In this technique, a specially designed tunneling device is introduced through the camera port and used to begin the formation of a subpleural tunnel.

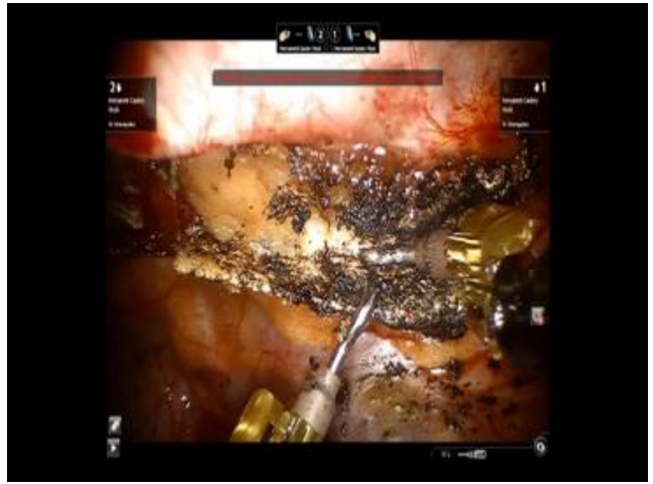

Figure 18 Phase IV-robotic dissection of the first rib and disarticulation of the SC joint. A 30-degree down viewing robotic camera is introduced through incision \#1. A hook cautery is placed in the right robotic arm in incision \#2, and a second hook cautery is placed in the left robotic arm in incision \#3. The hook in the left arm is used to put downward traction on the rib. SC, costo-sternal.

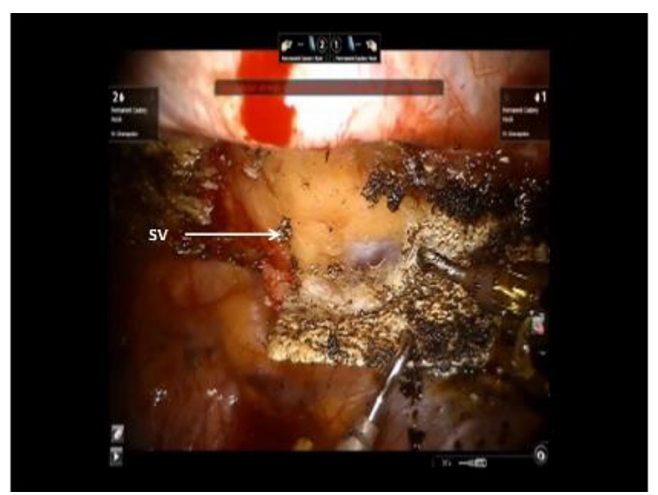

Figure 19 Phase IV-robotic dissection of the first rib and disarticulation of the SC joint, The hook cautery in the right robotic arm (30 cut/30 coagulation setting) is used to dissect the first rib away from the SV, disconnect the scalene muscles from the rib, and disarticulate the rib from the sternal and times clavicular joint. SC, costo-sternal; SV, subclavian vein.

After the formation of the tunnel, the metal tunneling device is withdrawn and a peelable sheath is positioned over the tunneler and replaced in the pleural tunnel. The metal tunneler is withdrawn and the sheath is left in place inside the pleural tunnel. Two 5 -inch On-Q soaker catheters are introduced through separate puncture sites placed anteriorly in the same IC space as the inferior incision. The On-Q soaker catheters are passed into the long subpleural sheath, the sheath is withdrawn and peeled away, leaving the soaker catheters in the subpleural tunnel. The catheters 


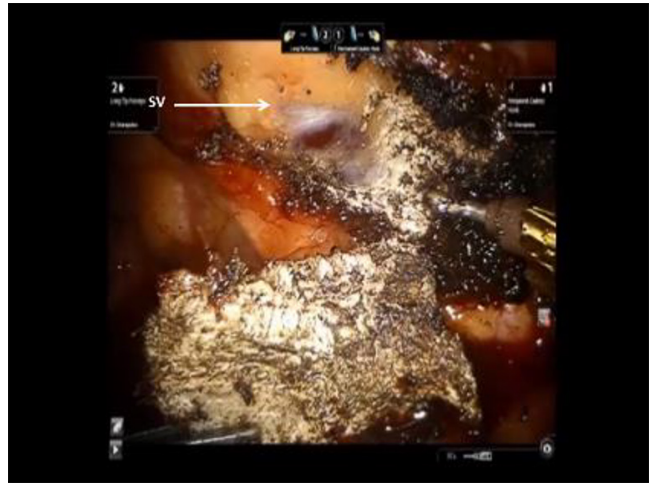

Figure 20 Phase IV-robotic dissection of the first rib and disarticulation of the SC joint. The SV is in very close proximity to the bone. $3 \mathrm{D}$ visualization aids in preventing injury to the SV. SC, costo-sternal; SV, subclavian vein; 3D, three-dimensional.

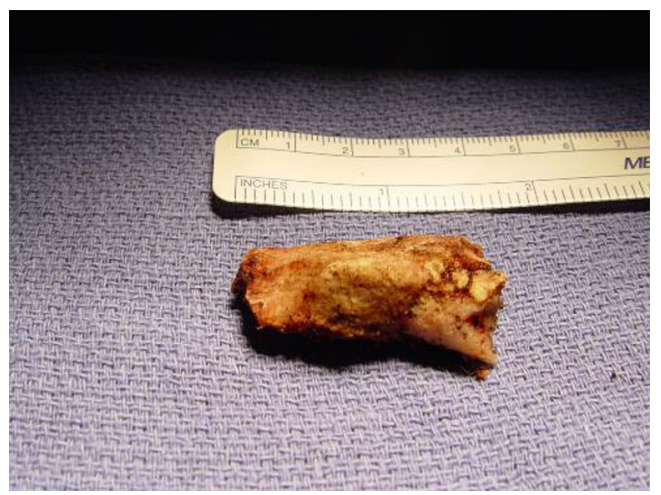

Figure 21 Resected "offending portion" of the congenitally malformed first rib in a patient with "neurogenic" TOS. The abnormal tubercle is clearly seen. TOS, thoracic outlet syndrome

are positioned in an overlapping staggered manner in order to provide infusion of the local anesthetic for the entirety of the pleural tunnel extending from the second to the 8th IC spaces. We use two catheters, an infusion of approximately $4 \mathrm{~mL} /$ hour $(2 \mathrm{~mL}$ per catheter) with a $400 \mathrm{~mL}$ reservoir with 0.125 bupivicaine. This system is used after the patient is discharged from the hospital, gives the patient 10 days of local pain control.

\section{Results}

A total of 162 patients have undergone robotic first rib resection by our group. Seventy-nine patients underwent robotic resection of the offending portion of the first rib for neurologic symptoms of the upper extremity (neurogenic
TOS). There were 29 men, 50 women. Mean age was $34 \pm 9.5$ years. Eighty-three patients underwent transthoracic robotic first rib resection for PSS. There were 49 men and 34 women. Mean age was $24 \pm 8.5$ years.

\section{Patients with neurogenic presentation (neurogenic TOS)}

Extrinsic compression of the SV by the medial aspect of the first rib was demonstrated on MRA in 75/79 (95\%) of patients with neurologic symptoms. In 5/79 (5\%) patients with neurologic symptoms, there was extrinsic compression of the SV and SA. Extrinsic compression of the SV by the medial aspect of the first rib was demonstrated on MRA with maneuvers in all patients with PSS.

Only the "offending portion" of first rib was removed. In all patients, a bony protuberance articulated with the underside of the clavicle just lateral to the sternocostal joint. Operative time was $87.6 \pm 10.8$ minutes. There were no intraoperative complications. Hospital stay ranged from 2 4 days with a median hospitalization of 3 days. There were no neurovascular complications. There was no mortality.

In patients with neurologic symptoms, Quick DASH Scores (mean \pm SEM) decreased from $60.3 \pm 2.1$ preoperatively to $5 \pm 2.3$ in the immediate postoperative period, and $3.5 \pm 1.1$ at 6 months $(\mathrm{P}<0.0001)$. Immediate relief of symptoms was seen in $71 / 79(91 \%)$ patients. Persistent paresthesia was seen in $9 / 79(9 \%)$ immediately postop and 3/79 (3.8\%) patients at 6 months. Two/seventynine $(2.5 \%)$ patients reported persistent symptoms of pain in the upper extremity at 6 months. Of interest these patients were among the oldest in the patient population and had experienced upper extremity pain for greater than 20 years. Seventy-seven/seventy-nine (97.4\%) of patients reported complete relief of symptoms. On postoperative MRA, there was relief of extrinsic compression of the $\mathrm{SV}$ in all patients.

\section{Patients with PSS (venous TOS)}

In these patients, the "offending portion" of first rib was removed. In all patients, a bony protuberance articulated with the underside of the clavicle just lateral to the sternocostal joint. Operative time was $127.6 \pm 20.8$ minutes. Median hospitalization was 4 days. There were no surgical complications, neurovascular injuries, or mortality.

In patients with PSS, $27 / 83$ patients $(31 \%)$ required endovascular venoplasty to completely open SV after the relief of the extrinsic compression. At 3, 6, 12 and 24 months, in all patients, MRA with maneuvers showed 
relief of extrinsic compression and patency of the SV. Two years after robotic resection of the offending portion of the first rib and obtaining patency of the SV, all patients remained asymptomatic and had full function of the affected upper extremity.

\section{Discussion}

Resection of the first rib has been performed by a high posterior thoracotomy, the transaxillary approach, the supraclavicular approach, the combined supraclavicular and infraclavicular approach, and thoracoscopy (28-34). High posterior thoracotomy was abandoned due to the associated morbidity and the adoption of the transaxillary approach which was described in the 1960's. The transaxillary approach which was originally described for the decompression of the brachial plexus and the SA, was first reported by Roos in 1966. Proponents of the transaxillary approach have asserted that it is less complicated than the supraclavicular or infraclavicular approaches as retraction of the brachial plexus and the vessels is not required. The supraclavicular approach is advocated by vascular surgeons. In patients with PSS, some surgeons have advocated the anterior infraclavicular approach combined with venoplasty and stent placement (34). However, the supraclavicular approach is hampered by the complexity of dissection around the brachial plexus and the vessels, and incomplete resection of the medial portion of the first rib. In general, the extrathoracic approaches to the first rib have been hampered by the potential of neurovascular complications and incomplete resection of the medial portion of the first rib.

Transthoracic thoracoscopic technique represented a minimally invasive approach to the first rib that could potentially obviate retraction of the neurovascular structures necessitated by the extrathoracic approaches (35). However, although this procedure was based on sound reasoning, it was limited by shortcomings of the conventional endoscopic instruments, and two-dimensional (2D) visualization.

Robotic surgical systems allow for high definition magnified $3 \mathrm{D}$ visualization of the operative field, are associated with accurate instrument maneuverability in a confined space, and may overcome the potential shortcomings of the conventional thoracoscopic approach.

Undoubtedly the use of robotic technology adds more ports, results in greater short term operative morbidity and longer hospitalization, longer operative times, and greater cost.

However, these shortcomings seem to be obviated by the greater accuracy of robotic dissection, the lower Incidence of neurovascular complications, excellent relief of symptoms in patients with "neurogenic TOS", and excellent long term patency of the SV and arm function in patients with PSS.

\section{Conclusions}

The excellent results which are associated with robotic resection of the "offending portion" of the first rib in patients with neurogenic and venous TOS, are due to the advantages of the robotic platform, as well as the greater understanding of the pathogenesis and appropriate diagnosis of TOS. Neurogenic and venous TOS appear to represent a different clinical manifestation of a congenital abnormality of the first rib at the SC joint. This congenital abnormality which by definition is bilateral with variable expression in the two upper extremities, results in extrinsic compression of the SV at its junction with the innominate vein by a bony tubercle at the SC joint. The compression of the subclavian-innominate junction in the thoracic outlet results in a spectrum of symptoms which range from neurologic symptoms resulting from venous ischemia of the upper extremity nerves (Peet's neurogenic TOS) to thrombosis of the SV caused by prolonged compression (PagetSchroetter or Peet's venous TOS). Therefore, neurogenic and venous TOS represent different clinical manifestations of the same disease and represent the spectrum from pain to venous thrombosis. It is suggested that these conditions are best classified "subclavian vein compression syndrome". MRA with maneuvers definitively identifies the extrinsic compression of the SV at its junction with the innominate vein and is diagnostic for this form of TOS. In turn, robotic transthoracic removal of the "offending portion" (the abnormal SC junction) of the first rib with preservation of the posterior aspect of the rib results in relief of the extrinsic compression and return of normal venous flow while obviating the neurologic complications that are associated with complete first rib resection and tranaxillary and supraclavicular techniques.

\section{Acknowledgments}

Funding: None.

\section{Footnote}

Provenance and Peer Review: This article was commissioned by the Guest Editor (Ghulam Abbas) for the series "Robotic 
Thoracic Surgery" published in Fournal of Thoracic Disease. The article has undergone external peer review.

Conflicts of Interest: All authors have completed the ICMJE uniform disclosure form (available at http://dx.doi. org/10.21037/jtd-2019-rts-04). The series "Robotic Thoracic Surgery" was commissioned by the editorial office without any funding or sponsorship. The authors have no other conflicts of interest to declare.

Ethical Statement: The authors are accountable for all aspects of the work in ensuring that questions related to the accuracy or integrity of any part of the work are appropriately investigated and resolved.

Open Access Statement: This is an Open Access article distributed in accordance with the Creative Commons Attribution-NonCommercial-NoDerivs 4.0 International License (CC BY-NC-ND 4.0), which permits the noncommercial replication and distribution of the article with the strict proviso that no changes or edits are made and the original work is properly cited (including links to both the formal publication through the relevant DOI and the license). See: https://creativecommons.org/licenses/by-nc-nd/4.0/.

\section{References}

1. Peet RM, Henriksen JD, Anderson TP, et al. Thoracicoutlet syndrome: evaluation of a therapeutic exercise program. Proc Staff Meet Mayo Clin 1956;31:281-7.

2. Atasoy E. History of thoracic outlet syndrome. Hand Clin 2004;20:15-6.

3. Blanchard B, Blanchard G, Forcier P, et al. The thoracic outlet: true syndromes, disputed syndrome (TOS, thoracic outlet syndrome). Rev Med Suisse Romande 1992;112:253-66.

4. Cooper A. An exostosis. In: Cooper A, Cooper B, Travers B. editors. Surgical Essays. 3rd ed. London: Longman, 1821:128.

5. Coote H. Exostosis of the left transverse process of the seventh cervical vertebra surrounded by blood vessels and nerves: successful removal. Lancet 1861;1:360-1.

6. Chang KZ, Likes K, Davis K, et al. The significance of cervical ribs in thoracic outlet syndrome. J Vasc Surg 2013;57:771-5.

7. Buzzard F. A case of cervical caries. Proc R Soc Med 1902;1:69.

8. Bramwell E, Dykes HB. Rib pressure and the brachial plexus. Edinburgh Med J 1927;27:65.

9. Murphy T. Brachial plexus neuritis caused by pressure of the first rib. Aust Med J 1910;15:582-5.

10. Ochsner A, Gage M, Debakey M. Scalenus anticus syndrome. Am J Surg 1935;28:669-95.

11. Falconer MA, Weddell G. Costoclavicular compression of the subclavian artery and vein. Lancet 1943;2:539-43.

12. Adson AW, Coffey JR. Cervical rib: a method of anterior approach for relief of symptoms by division of the scalenus anticus. Ann Surg 1927;85:839-57.

13. Adson AW. Surgical treatment for symptoms produced by cervical ribs and the scalenus anticus muscle. Surg Gynecol Obstet 1947;85:687-700.

14. Hixson KM, Horris HB, McLeod TCV, et al. The diagnostic accuracy of clinical diagnostic tests for thoracic outlet syndrome. J Sport Rehabil 2017;26:459-65.

15. Paget J. Clinical lectures and essays. London: Longmans, Green \& Co, 1875.

16. von Schroetter L. Nathnagel Handbuch der Pathologie und Therapie, Anonymous Wein, Holder. 1884.

Erkrankungen der Gefasse.

17. Hughes ES. Venous obstruction in upper extremity. Br J Surg 1948; 36:155-63.

18. Illig KA, Doyle AJ. A comprehensive review of PagetSchroetter syndrome. J Vasc Surg 2010;51:1538-47.

19. Peek J, Vos CG, Ünlü Ç, et al. Long-term functional outcome of surgical treatment for thoracic outlet syndrome. Diagnostics (Basel) 2018;8:7.

20. Peek J, Vos CG, Ünlü Ç, et al. Outcome of surgical treatment for thoracic outlet syndrome: systematic review and meta-analysis. Ann Vasc Surg 2017;40:303-26.

21. Gharagozloo F, Meyer M, Tempesta B, et al. Proposed pathogenesis of Paget-Shroetter disease: impingement of the subclavian vein by a congenitally malformed boney tubercle on the first rib. J Clin Pathol 2012;65:262-6.

22. Gharagozloo F, Meyer M, Tempesta B, et al. Robotic en bloc first-rib resection for Paget-Schroetter disease, a form of thoracic outlet syndrome: technique and initial results. Innovations 2012;7:39-44.

23. Gharagozloo F, Meyer M, Tempesta B, et al. Robotic transthoracic first-rib resection for Paget-Schroetter syndrome. Eur J Cardiothorac Surg 2019;55:434-9.

24. Kobayashi S, Takeno K, Miyazaki T, et al. Effects of arterial ischemia and venous congestion on the lumbar nerve root in dogs. J Orthop Res 2008;26:1533-40.

25. Gummesson C, Ward MM, Atroshi I. The shortened disabilities of the arm, shoulder and hand questionnaire (QuickDASH): validity and reliability based on responses 
within the full-length DASH. BMC Musculoskelet Disord 2006;7:44.

26. Matheson LN, Melhorn M, Mayer TG, et al. Reliability of a visual analog version of the Quick DASH. J Bone Joint Surg Am 2006;88:1782-7.

27. Molina JE, Hunter DW, Dietz CA. Protocols for Paget-Schroetter Syndrome and late treatment of chronic subclavian vein obstruction. Ann Thorac Surg 2009;87:416-22.

28. Gloviczki P, Kazmier FJ, Hollier LH. Axillary-subclavian venous occlusion: the morbidity of a nonlethal disease. J Vasc Surg 1986;4:333-7.

29. DeLee JC, Drez D Jr, Miller MD, et al. Shoulder. In: DeLee JC, Drez D Jr, Miller MD. editors. DeLee and Drez's Orthopaedic Sports Medicine. 3rd ed. Philadelphia: Elsevier Saunders, 2009:chap 17.

30. Clagett OT. Presidential address, American Association of Thoracic Surgery Research and Prosearch. J Thorac

Cite this article as: Gharagozloo F, Atiquzzaman N, Meyer M, Tempesta B, Werden S. Robotic first rib resection for thoracic outlet syndrome. J Thorac Dis 2021;13(10):6141-6154. doi: 10.21037/jtd-2019-rts-04
Cardiovasc Surg 1962;44:153-66.

31. Loscertales J, Congregado M, Jiménez Merchán R. First rib resection using videothorascopy for the treatment of thoracic outlet syndrome. Arch Bronconeumol 2011;47:204-7.

32. Roos DB. Transaxillary approach for first rib resection to relieve thoracic outlet syndrome. Ann Surg 1966;163:354-8.

33. Urschel HC Jr, Patel AN. Thoracic Outlet Syndromes. In: Kaiser LR, Kron IL, Spray TL. editors. Mastery of Cardiothoracic Surgery. Philadelphia: Lippincott Williams \& Wilkins, 2007:213.

34. Hempel GK, Shutze WP, Anderson JF, et al. 770 consecutive supraclavicular first rib resections for thoracic outlet syndrome. Ann Vasc Surg 1996;10:456-63.

35. George RS, Milton R, Chaudhuri N, et al. Totally endoscopic (VATS) first rib resection for thoracic outlet syndrome. Ann Thorac Surg 2017;103:241-5. 